\title{
Model Based Research of Dynamic Performance of Shaft-Bearing System in High-Speed Field
}

\author{
Teng Hu, Guofu Yin, and Mingnan Sun \\ School of Manufacturing Science and Engineering, Sichuan University, Chengdu, Sichuan 610065, China \\ Correspondence should be addressed to Teng Hu; hu.t.scu@gmail.com
}

Received 12 May 2013; Revised 18 February 2014; Accepted 19 February 2014; Published 17 April 2014

Academic Editor: Valder Steffen

Copyright (C) 2014 Teng Hu et al. This is an open access article distributed under the Creative Commons Attribution License, which permits unrestricted use, distribution, and reproduction in any medium, provided the original work is properly cited.

\begin{abstract}
Dynamic performance of the high-speed running shaft-bearing system (SBS) is different from that of idle state system due to the high-speed effects (HSE), including shaft centrifugal force, gyroscopic moment, and nonlinear bearing operational stiffness. In this paper, aiming at improving the operation stability, dynamic performance of SBS operating in high-speed field is investigated based on a finite element (FE) dynamic model. Firstly, the Timoshenko beam elements are applied to develop the SBS FE model with full consideration of HSE. Secondly, idle state frequency response function at the front tip is obtained analytically and experimentally to validate that the FE model can illustrate the system dynamic behaviors in static condition. Finally, by substituting various rotational velocities into the FE model, the HSE on system natural frequencies are studied one by one as well as together. The results show that, when bearing is being extremely light preloaded, SBS frequencies are affected by the HSE of shaft more than bearing, especially where the gyroscopic moment effect of shaft is the most influential factor. Moreover, the nonmonotonic variation of bearing operational stiffness is analyzed. The "stiffen" phenomenon explained in this paper provides a more comprehensive understanding of the nonlinear bearing operational stiffness.
\end{abstract}

\section{Introduction}

High-speed machining technique (HSM) is highly developed recently due to the remarkable requirement of machining efficiency in such industrial fields like aerospace, military, vehicle, energy, and so forth. High-speed spindle (HSS) system, whose dynamic performance is the key factor to evaluate HSM level, is regarded as the main carrier of HSM. While HSS operates at low speed, its dynamic behaviors can be scarcely affected by centrifugal forces and gyroscopic moments, and the bearing stiffness remains constant. Thus in this situation, these speed-related factors are ignored. However, when operating speed is extraordinarily high, the centrifugal forces and gyroscopic moments become so strong and the bearing stiffness changes a great deal. Diversity of the HSS dynamic performance due to the varying operation speed manifests that the low-speed (or stationary) dynamic behavior is insufficient to describe the characteristics of HSS when high-speed effects are taken into account. Here, we define the phrases high-speed effects for the centrifugal forces and gyroscopic moments of shaft and the nonlinear bearing stiffness. Therefore, it is scientifically and strategically significant to build the speed-varying dynamic model in order to further predict the HSS high-speed performance.

A typical HSS can be simply regarded as a shaft-bearing system (SBS), since the high-speed effects are induced by rotational velocity and SBS is the primary rotating module of HSS.

The most important option during the procedure of investigating the SBS dynamic performance is to create the nonlinear model of bearing stiffness, which is dependent on rotating speed. In 1950s-1960s, the static and the quasidynamic analysis models of bearing were proposed by Palmgren $[1]$ and Jones [2, 3], respectively. Harris's model [4] then became the most popular one dealing with nonlinear bearing analysis which was developed and improved on the basis of Jones's model. Although Gupta [5] introduced another bearing dynamic analysis approach, it was actually less 


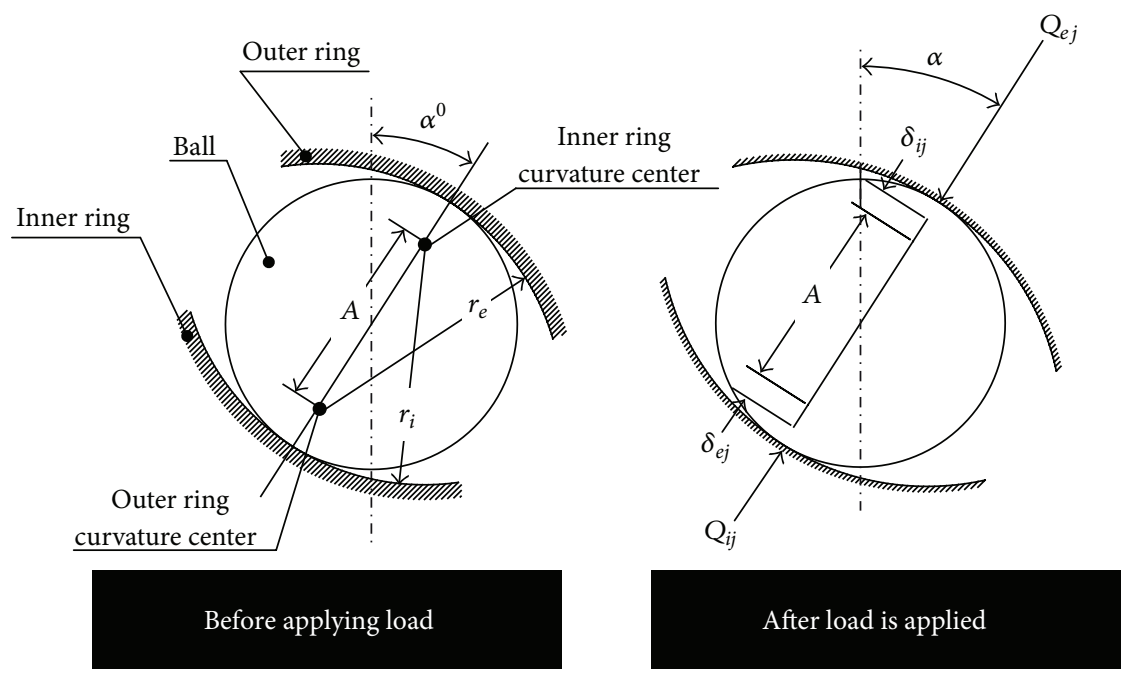

FIGURE 1: Relationship between normal contact load and deformation.

instructional due to the extraordinarily complex kinematics principle of rolling bearing. In recent years, Liao and Lin [6] established a 3D expression for the elastic deformation at different azimuth in terms of the contact geometry surface at the rings. Several characteristics due to the varying contact angle were discussed too. Zverv et al. [7] investigated the elastic issue of rolling bearing under high-speed operation and heavy preload condition. And bearing selection criterion during the design stage of spindle unit was provided. The Hertz contact problem was studied by Kang et al. [8] using finite element method and curve fitting technology with a modified Harris model. Besides, as is presented in [9], the artificial neutral network model is implemented to calculate the operational stiffness of rolling bearings. Jedrzejewski and Kwasny [10] employed Harris's model as well to analyze the contact stiffness and deformation of angular contact ball bearings, both of which were affected by centrifugal forces and gyroscopic moments of the rolling balls. The bearing stiffness was written in matrix form by Guo and Parker [11] based on the research of different rolling bearings by combining finite element method (FEM) and contact mechanics. By introducing the contact mechanism into the FEM, Guo and Parker [11] presented the matrix form of the bearing stiffness for the first time.

On the other hand, many studies have been conducted to model the shaft dynamic behaviors. Nelson [12] generalized the research work before 1970s and built the shaft dynamic finite element model by utilizing the Timoshenko beam theory instead of the Euler beam theory, thereby including transverse shear effect. An integrated thermal mechanical dynamic model with linear bearing stiffness was constructed by Lin et al. [13] based on Timoshenko beam theory and FEM. Ertürk et al. [14] compared the dynamics of spindletool system by employing the Euler-Bernoulli beam theory and the Timoshenko beam theory. The comparison indicated that the Euler-Bernoulli beam is inadequate for modeling the rotor system within high frequency range. The receptance coupling technique [15] was also adopted in their work to identify the frequency response function (FRF) of tool tip with linear bearing stiffness. Jiang and Zheng [16] used transfer matrix method (TMM) to model the spindle unit, and its bearing model was also based on Harris's theory. Chen and Hwang [17] used Lagrange's approach to study the motorized high-speed spindle dynamics, but the centrifugal effect was the only speed-related consideration. Wang et al. [18] completed an identification of tool-spindle interface FRF, yet their work only used the Euler-Bernoulli beam theory to model the spindle system, where the high-speed effects were excluded.

Apparently high-speed field has a strong influence on SBS. However, little research has been conducted to predict the dynamic performance of SBS at high speeds. In this paper, a SBS dynamic model is presented based on the Timoshenko beam theory and FEM. The nonlinear bearing analysis model is developed using Harris's methodology. And the nonmonotonic radial operational stiffness of angular contact ball bearing is calculated. Integrating the beam and bearing model, the FRF at the front tip is obtained. The proposed FE model is verified by impact tests with the modal test facilities; high-speed effects are then comprehensively predicted and studied based on the validated FE model.

\section{Dynamic Modeling of the SBS}

\subsection{Nonlinear Model of Angular Contact Ball Bearing}

\subsubsection{Hertzian Contact of Angular Contact Ball Bearing.} Angular contact ball bearing stiffness can be regarded as the tandem results of contact stiffness between rolling elements and rings. Generally, as depicted in Figure 1, based on the Hertzian contact theory, the contact relationships between rolling elements and rings are

$$
Q_{i j}=k_{i j} \delta_{i j}^{1.5} \quad Q_{e j}=k_{e j} \delta_{e j}^{1.5},
$$




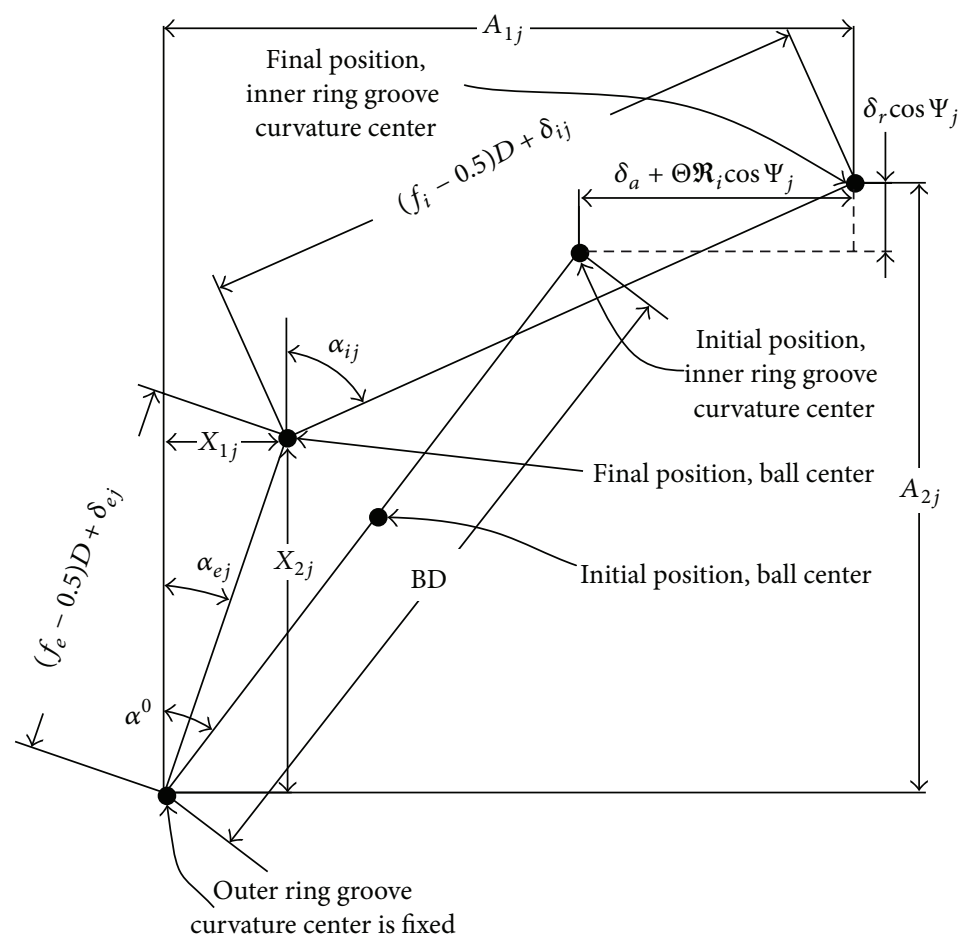

FIGURE 2: Deformed relationship between rolling element and rings.

where $k_{i j}$ and $k_{e j}$ are contact coefficients. And the contact stiffness, $K_{i j}^{B}$ and $K_{e j}^{B}$, can be obtained through derivation calculus to (1) as follows:

$$
K_{i j}^{B}=1.5 k_{i j} \delta_{i j}^{0.5} \quad K_{e j}^{B}=1.5 k_{e j} \delta_{e j}^{0.5} .
$$

\subsubsection{Deformation and Contact Force of Angular Contact Ball} Bearing. If the bearing remains in the no-load and static state, the centers of curvature of the inner and outer rings grooves and the center of rolling element will be collinear. When bearing is under high-speed operation, contact angle between inner ring and rolling element will increase and contact angle between outer ring and rolling element will decrease duo to the centrifugal effect, for it can be assumed that the curvature center of outer ring is fixed; hence the deformed relationship of bearing is presented in Figure 2 . Consequently, the following equation can be derived from Figure 2:

$$
\begin{gathered}
\cos \alpha_{e j}=\frac{X_{2 j}}{\Delta_{e j}}=\frac{X_{2 j}}{\left(f_{e}-0.5\right) D+\delta_{e j}}, \\
\sin \alpha_{e j}=\frac{X_{1 j}}{\Delta_{e j}}=\frac{X_{1 j}}{\left(f_{e}-0.5\right) D+\delta_{e j}}, \\
\cos \alpha_{i j}=\frac{A_{2 j}-X_{2 j}}{\Delta_{i j}}=\frac{A_{2 j}-X_{2 j}}{\left(f_{i}-0.5\right) D+\delta_{i j}}, \\
\sin \alpha_{i j}=\frac{A_{1 j}-X_{1 j}}{\Delta_{i j}}=\frac{A_{1 j}-X_{1 j}}{\left(f_{i}-0.5\right) D+\delta_{i j}},
\end{gathered}
$$

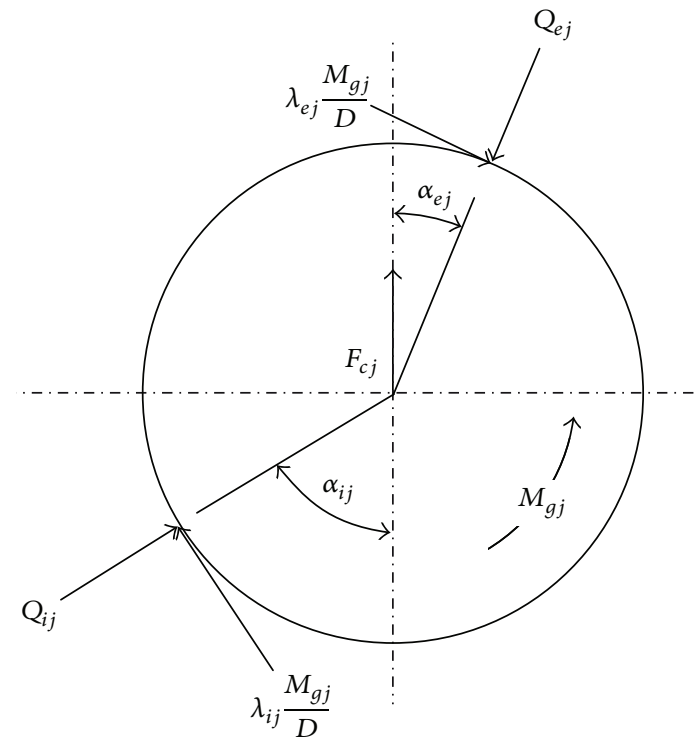

FIGURE 3: Mechanical analysis of rolling element.

$$
\begin{gathered}
\left(A_{1 j}-X_{1 j}\right)^{2}+\left(A_{2 j}-X_{2 j}\right)^{2}-\left[\left(f_{i}-0.5\right) D+\delta_{i j}\right]^{2}=0, \\
X_{1 j}^{2}+X_{2 j}^{2}-\left[\left(f_{i}-0.5\right) D+\delta_{i j}\right]^{2}=0 .
\end{gathered}
$$

Mechanical analysis of a single rolling element is presented in Figure 3, where centrifugal force and gyroscopic 


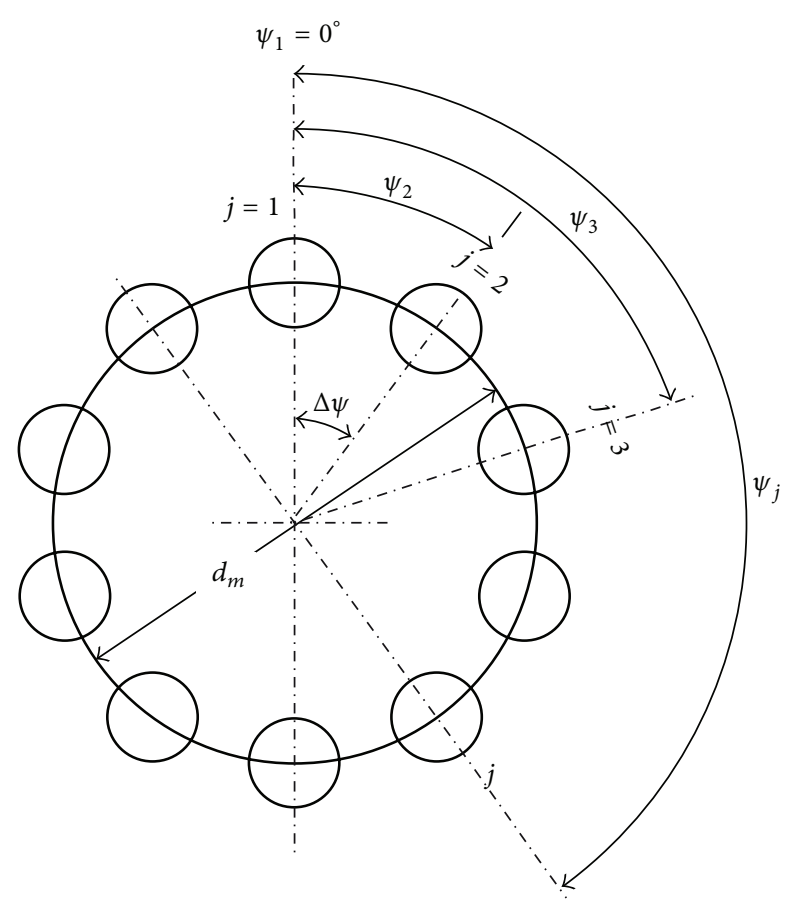

FIGURE 4: Azimuth of rolling bearing.

moment are considered simultaneously. The following equations can be derived then by combining the azimuth of bearing shown in Figure 4:

$$
\begin{aligned}
& Q_{i j} \sin \alpha_{i j}-Q_{e j} \sin \alpha_{e j}-\frac{M_{g j}}{D}\left(\lambda_{i j} \cos \alpha_{i j}-\lambda_{e j} \cos \alpha_{e j}\right)=0, \\
& Q_{i j} \cos \alpha_{i j}-Q_{e j} \cos \alpha_{e j}-\frac{M_{g j}}{D}\left(\lambda_{i j} \sin \alpha_{i j}-\lambda_{e j} \sin \alpha_{e j}\right) \\
& \quad+F_{c j}=0,
\end{aligned}
$$

where $\lambda_{i j}=\lambda_{e j}=1$ for inner ring control; $\lambda_{i j}=0$ and $\lambda_{e j}=2$ for outer ring control. The centrifugal force and gyroscopic moment of a single rolling element can be expressed as

$$
\begin{gathered}
F_{c}=\frac{1}{2} m d_{m} \omega_{m}^{2}, \\
M_{g j}=J\left(\frac{\omega_{R}}{\omega}\right)_{j}\left(\frac{\omega_{m}}{\omega}\right)_{j} \omega^{2} \sin \beta,
\end{gathered}
$$

where $J$ is the rolling element mass moment of inertia.

External force acting on the inner ring (or outer ring) of bearing should be equal to the sum of forces acting on rolling elements in axial direction and radial direction, respectively. Thus, consider the following:

$$
\begin{aligned}
& F_{a}-\sum_{j=1}^{j=Z}\left(Q_{i j} \sin \alpha_{i j}-\frac{\lambda_{i j} M_{g j}}{D} \cos \alpha_{i j}\right)=0, \\
& F_{r}-\sum_{j=1}^{j=Z}\left(Q_{i j} \cos \alpha_{i j}+\frac{\lambda_{i j} M_{g j}}{D} \sin \alpha_{i j}\right) \cos \psi_{j}=0,
\end{aligned}
$$

$$
\begin{gathered}
M-\sum_{j=1}^{j=Z}\left[\left(Q_{i j} \sin \alpha_{i j}-\frac{\lambda_{i j} M_{g j}}{D} \sin \alpha_{i j}\right) \mathfrak{R}_{i}\right. \\
\left.+\frac{\lambda_{i j} M_{g j}}{D} r_{i}\right] \cos \psi_{j}=0 .
\end{gathered}
$$

Knowing the specific rotating speed, axial load, and radial load as input conditions and giving initial value to $\delta_{a}, \delta_{r}$, $X_{1 j}, X_{2 j}, \delta_{i j}$, and $\delta_{e j}$, simultaneous nonlinear equations (3)(6) can be solved numerically by using the Newton-Raphson method. Thus $\alpha_{i j}, \alpha_{e j}, \delta_{i j}, \delta_{e j}, Q_{i j}$, and $Q_{e j}$ are calculated iteratively, and contact stiffness of bearing can be obtained through (2).

2.1.3. Stiffness Matrix of Angular Contact Ball Bearing. Under the operation state, external loads, that is, the axial force, radial force, and moment act on angular contact ball bearing simultaneously. It is important to understand that deformations of both bearing inner and outer rings are the coupling results of different external loads. Therefore, bearing stiffness can be given in the following matrix form:

$$
\mathbf{K}_{\mathrm{CB}}=\left[\begin{array}{ll}
\mathbf{K}_{I I} & \mathbf{K}_{I E} \\
\mathbf{K}_{E I} & \mathbf{K}_{E E}
\end{array}\right]
$$

where $\mathbf{K}_{I I}$ and $\mathbf{K}_{I E}$ represent the contribution of inner ring stiffness term and outer ring stiffness term, respectively, due to the external loads that act on inner ring. $\mathbf{K}_{E I}$, and $\mathbf{K}_{E E}$ have the similarly corresponding meaning, respectively.

Assume that external loads acting on bearing include axial force $F_{x}$, radial forces $F_{y}$ and $F_{z}$, and moments $M_{y}$ and $M_{z}$ and that vector of bearing ring displacement is $\left[\delta_{I x}, \delta_{I y}, \delta_{I z}, \gamma_{I y}, \gamma_{I z} ; \delta_{E x}, \delta_{E y}, \delta_{E z}, \gamma_{E y}, \gamma_{E z}\right]^{T}$, where $\delta$ and $\gamma$ are the translational and rotational displacements, respectively. For the inner ring of bearing, equation (6) can be extended as the following equation:

$$
\begin{gathered}
F_{x}^{I}=\sum_{j=1}^{j=Z}\left(Q_{i j} \sin \alpha_{i j}-\frac{\lambda_{i j} M_{g j}}{D} \cos \alpha_{i j}\right), \\
F_{y}^{I}=\sum_{j=1}^{j=Z}\left(Q_{i j} \cos \alpha_{i j}+\frac{\lambda_{i j} M_{g j}}{D} \sin \alpha_{i j}\right) \cos \psi_{j}, \\
F_{z}^{I}=\sum_{j=1}^{j=Z}\left(Q_{i j} \cos \alpha_{i j}+\frac{\lambda_{i j} M_{g j}}{D} \sin \alpha_{i j}\right) \sin \psi_{j}, \\
M_{y}^{I}=\sum_{j=1}^{j=Z}\left[\left(Q_{i j} \sin \alpha_{i j}-\frac{\lambda_{i j} M_{g j}}{D} \sin \alpha_{i j}\right) \Re_{i}\right. \\
\left.+\frac{\lambda_{i j} M_{g j}}{D} r_{i}\right] \cos \psi_{j},
\end{gathered}
$$




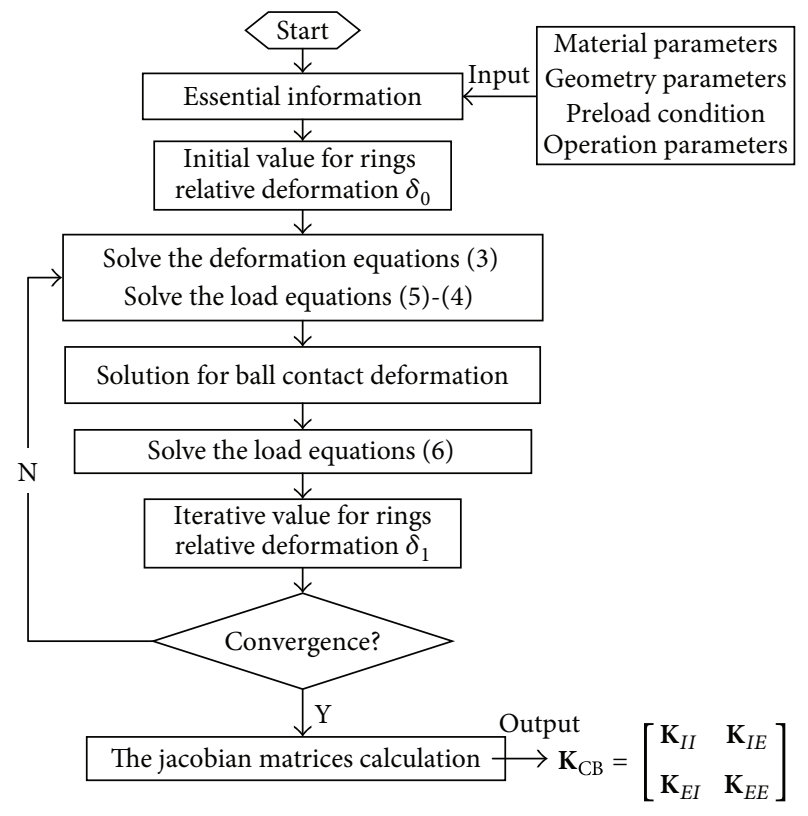

FIGURE 5: Flow chart of angular contact ball bearing stiffness matrix calculation procedure.

$$
\begin{gathered}
M_{z}^{I}=-\sum_{j=1}^{j=Z}\left[\left(Q_{i j} \sin \alpha_{i j}-\frac{\lambda_{i j} M_{g j}}{D} \sin \alpha_{i j}\right) \Re_{i}\right. \\
\left.+\frac{\lambda_{i j} M_{g j}}{D} r_{i}\right] \sin \psi_{j} .
\end{gathered}
$$

Using the iterative results of Section 2.1.2, $\mathbf{K}_{I I}$ can be calculated by the derivatives of external loads with respect to displacements of inner ring as the Jacobian matrix form:

$$
\mathbf{K}_{I I}=\left[\begin{array}{ccccc}
\frac{\partial F_{x}^{I}}{\partial \delta_{I x}} & \frac{\partial F_{x}^{I}}{\partial \delta_{I y}} & \frac{\partial F_{x}^{I}}{\partial \delta_{I z}} & \frac{\partial F_{x}^{I}}{\partial \gamma_{I y}} & \frac{\partial F_{x}^{I}}{\partial \gamma_{I z}} \\
\frac{\partial F_{y}^{I}}{\partial \delta_{I x}} & \frac{\partial F_{y}^{I}}{\partial \delta_{I y}} & \frac{\partial F_{y}^{I}}{\partial \delta_{I z}} & \frac{\partial F_{y}^{I}}{\partial \gamma_{I y}} & \frac{\partial F_{y}^{I}}{\partial \gamma_{I z}} \\
\frac{\partial F_{z}^{I}}{\partial \delta_{I x}} & \frac{\partial F_{z}^{I}}{\partial \delta_{I y}} & \frac{\partial F_{z}^{I}}{\partial \delta_{I z}} & \frac{\partial F_{z}^{I}}{\partial \gamma_{I y}} & \frac{\partial F_{z}^{I}}{\partial \gamma_{I z}} \\
\frac{\partial M_{y}^{I}}{\partial \delta_{I x}} & \frac{\partial M_{y}^{I}}{\partial \delta_{I y}} & \frac{\partial M_{y}^{I}}{\partial \delta_{I z}} & \frac{\partial M_{y}^{I}}{\partial \gamma_{I y}} & \frac{\partial M_{y}^{I}}{\partial \gamma_{I z}} \\
\frac{\partial M_{z}^{I}}{\partial \delta_{I x}} & \frac{\partial M_{z}^{I}}{\partial \delta_{I y}} & \frac{\partial M_{z}^{I}}{\partial \delta_{I z}} & \frac{\partial M_{z}^{I}}{\partial \gamma_{I y}} & \frac{\partial M_{z}^{I}}{\partial \gamma_{I z}}
\end{array}\right] .
$$

Similarly, $\mathbf{K}_{I E}, \mathbf{K}_{E I}$, and $\mathbf{K}_{E E}$ can be deduced. Figure 5 represents the general calculation procedure of stiffness matrix $\mathbf{K}_{\mathrm{CB}}$ of angular contact ball bearing.

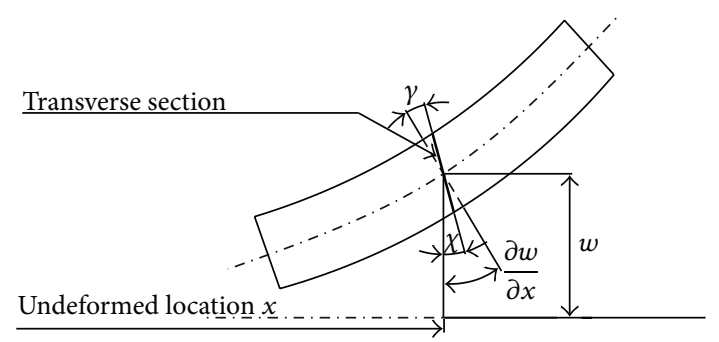

Figure 6: The Timoshenko beam kinematics.

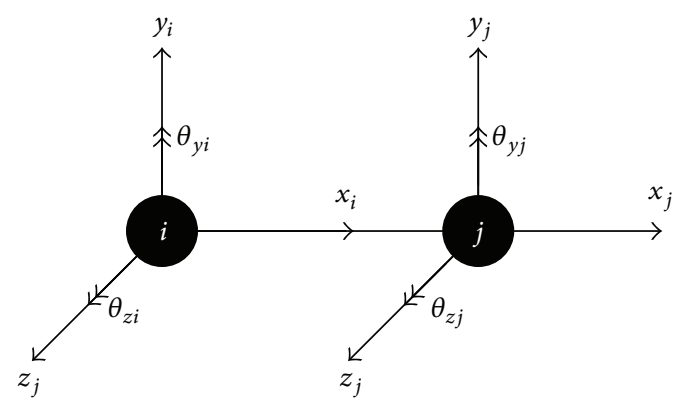

FIGURE 7: Reference frame of the typical beam element.

\subsection{Dynamic Modeling SBS Based on the Timoshenko Beam Theory and Finite Element Method}

2.2.1. Dynamic Model of Beam Element. Components like shaft, bearing housing, and motor stator of SBS are modeled as beam element. According to the classical Euler-Bernoulli beam theory, even though shear loads act on transverse section of beam, there is no shear strain [19]. The Timoshenko beam theory, however, regarded as correction and improvement of the Euler-Bernoulli beam theory, incorporates the rotating inertia of transverse section into governing equation of beam (Figure 6). It is reasonable and accurate enough to simulate the dynamic performance of high-speed rotor system [12]. In addition, although finite element method is not the best solution against the dynamic problems, it is of brilliant accuracy to extract the lower order eigenvalue and eigenvector of beam structure [20].

Figure 7 shows a typical Timoshenko beam element with 2 nodes $(i, j)$, where $X$ direction is the axial direction of beam element. Each node has 3 translational degrees of freedom and 2 rotational degrees of freedom. The displacement vector of element can be written as

$$
\{q\}=\left\{x_{i}, y_{i}, z_{i}, \theta_{y i}, \theta_{z i}, x_{j}, y_{j}, z_{j}, \theta_{y_{j}}, \theta_{z j}\right\} .
$$

By ignoring the damping and using the FEM, equation of motion for the Timoshenko beam element is expressed as the following matrix form:

$$
\begin{aligned}
& {\left[M_{\mathrm{bm}}\right]\{\ddot{q}\}-\Omega\left[G_{\mathrm{bm}}\right]\{\dot{q}\}} \\
& \quad+\left(\left[K_{\mathrm{bm}}\right]-\Omega^{2}\left[M_{\mathrm{bc}}\right]\right)\{q\}=\left\{F_{\mathrm{bm}}\right\}
\end{aligned}
$$




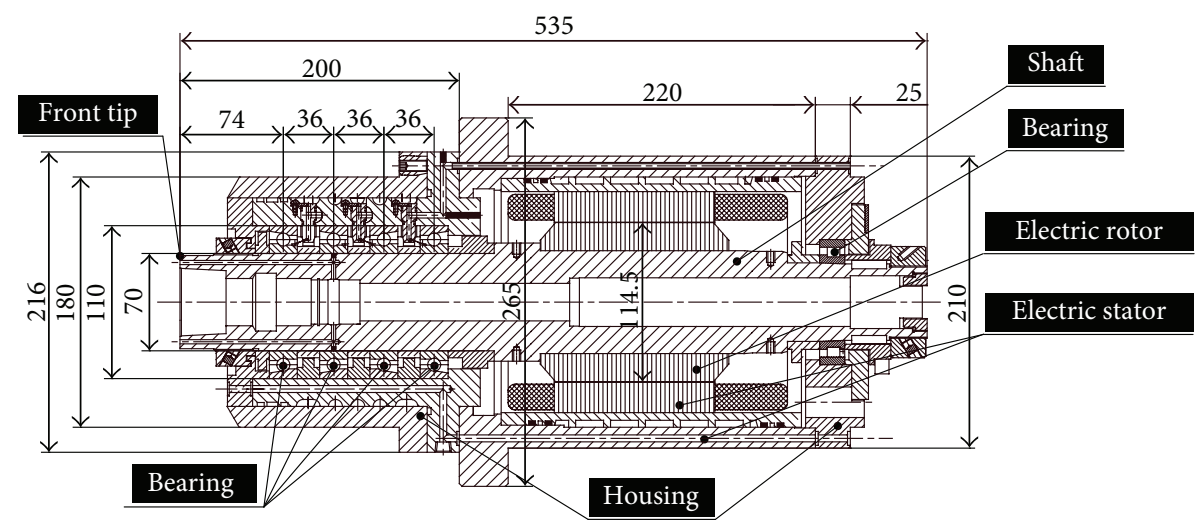

FIGURE 8: Sketch of the SBS.

TABLE 1: Basic parameters of 70BNR10X.

\begin{tabular}{lccccccc}
\hline Inner diameter & Outer diameter & Thickness & Mass & Ball diameter & $\begin{array}{c}\text { Groove curvature } \\
\text { radius coefficient }\end{array}$ & Number of balls & Contact angle \\
\hline $70 \mathrm{~mm}$ & $110 \mathrm{~mm}$ & $20 \mathrm{~mm}$ & $0.605 \mathrm{~kg}$ & $8.731 \mathrm{~mm}$ & 0.53 & 25 & $18^{\circ}$ \\
\hline
\end{tabular}

where $\left[M_{\mathrm{bm}}\right],\left[G_{\mathrm{bm}}\right]$, and $\left[K_{\mathrm{bm}}\right]$ are the mass matrix, gyroscopic moment matrix, and the stiffness matrix of beam element, respectively. $\left[M_{\mathrm{bc}}\right]$ is the centrifugal-related mass matrix, and $F_{\mathrm{bm}}$ is the force vector.

2.2.2. Dynamic Model of Disk Element. Components like motor rotor and bearing spacer of SBS are treated as disk elements. By using FEM as well, their equation of motion can be expressed as follows:

$$
\left[M_{\text {disk }}\right]\{\ddot{q}\}-\Omega\left[G_{\text {disk }}\right]\{\dot{q}\}=\left\{F_{\text {disk }}\right\},
$$

where $\left[M_{\text {disk }}\right]$ is the mass matrix, $\left[G_{\text {disk }}\right]$ is the gyroscopic moment matrix, and $\left\{F_{\text {disk }}\right\}$ is the force vector.

2.2.3. Finite Element Dynamic Model of SBS. By coupling the local dynamics of bearing with the global beam and disk motion, the speed-varying dynamic equation of SBS is finally obtained as follows:

$$
[M]\{\ddot{u}\}+[C]\{\dot{u}\}+[K]\{u\}=\{F\},
$$

where $[M]$ is the mass matrix of system, $[M]=\left[M_{\mathrm{bm}}\right]+$ $\left[M_{\text {disk }}\right]$. $[K]$ is the equivalent stiffness matrix of system, $[K]=$ $\left[K_{\mathrm{bm}}\right]+\left[K_{\mathrm{bpa}}\right]-\Omega^{2}\left[M_{\mathrm{bc}}\right]+\left[K_{\text {bearing }}\right]$, where $K_{\mathrm{bpa}}$ is the stiffness matrix due to the axial force. $[C]$ is the equivalent damping matrix of system, $[C]=\left[C_{\text {system }}\right]-\Omega\left[G_{\text {disk }}\right]-$ $\Omega\left[G_{\mathrm{bm}}\right]$. And $\left[C_{\text {system }}\right]$, which is estimated from impact test, is the system structural damping. In this paper, the Rayleigh damping is employed. Hence, $\left[C_{\text {system }}\right]$ is related to $[M]$ and $[K]$ as follows:

$$
\left[C_{\text {system }}\right]=\left(\alpha+\alpha_{c}\right)[K]+\beta[M],
$$

where $\alpha, \beta$, and $\alpha_{c}$ are the constants calculated as follows:

$$
\begin{gathered}
\alpha=\frac{2\left(\xi_{2} f_{2}-\xi_{1} f_{1}\right)}{\left(f_{2}^{2}-f_{1}^{2}\right)}, \\
\beta=\frac{2 f_{1} f_{2}\left(\xi_{1} f_{2}-\xi_{2} f_{1}\right)}{\left(f_{2}^{2}-f_{1}^{2}\right)}, \\
\alpha_{c}=\frac{\xi_{\text {system }}}{\pi f},
\end{gathered}
$$

where $f_{1}$ and $f_{2}$ are two different natural frequencies and $\xi_{1}$ and $\xi_{2}$ are system damping ratios at $f_{1}$ and $f_{2}$, respectively. $\xi_{\text {system }}$ is the structural damping ratio. $f$ is the excitation frequency.

Once $f_{1}, f_{2}, \xi_{1}$, and $\xi_{2}$ are derived from impact test and $\xi_{\text {system }}$ is assigned, $\left[C_{\text {system }}\right]$ is obtained according to (14)-(15).

\section{Verification of the Proposed SBS Dynamic Model}

3.1. Prototype of SBS. The SBS FE model is validated through impact test against a prototype ZZD63200 which has a maximum speed of $20000 \mathrm{rpm}$. Structure sketch and basic dimensions of the system are shown in Figure 8. Four NSK high-speed angular contact ceramic ball bearings 70BNR10X, structural parameters of which can be found in Table 1, are arranged in double back-to-back configuration to form the front bearing set. The front bearing set is initially preloaded via fixed position preload mechanism with an extremely light (EL) preload of $285 \mathrm{~N}$. Thus, the bearing preload state is achieved by adjusting the clearance between inner ring and outer ring during the assemblage process. Rear bearing set of the system consists of only one NSK roller bearing N1011RXTPKR. 


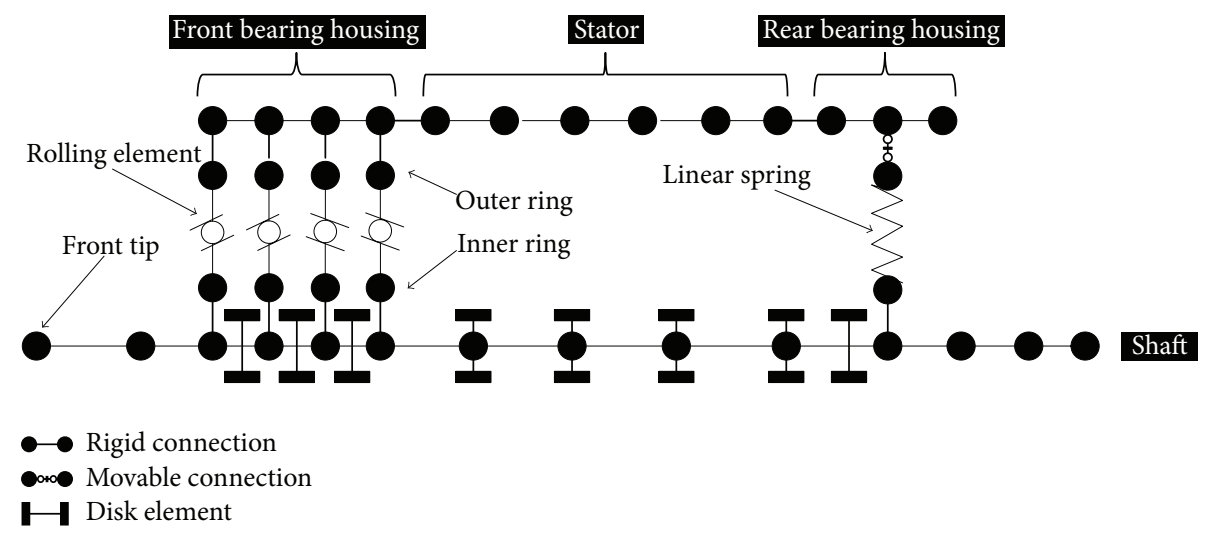

FIGURE 9: Finite element model of the SBS.

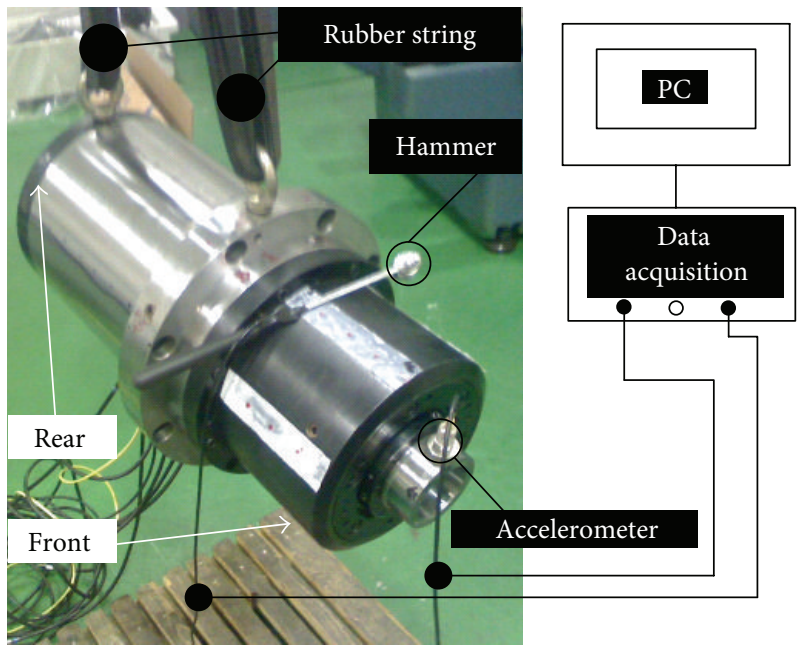

FIGURE 10: Scene of impact test based on LMS Test Lab.

Features like chamfer or screw thread should be ignored in FE model in order to speed up the calculation. Nodes of the FE model should be located in bearing positions and in positions where section dimensions change evidently. Finally the FE model is shown in Figure 9.

3.2. Verification of the SBS FE Model. As is shown in Figure 10, the SBS prototype is hung by rubber strings to realize an approximate free-free boundary condition. The direct and cross FRFs at the front tip are extracted by performing the dynamic experimentation with impact testing module of LMS Test Lab. The simulation and experimentation results are presented in Figure 11, through which the reliability of the proposed SBS FE model is validated. And more details can be found in Table 2.

\section{Prediction of the Dynamic Performance of SBS in High-Speed Field}

The high-speed effects, including centrifugal effect of shaft, gyroscopic effect of shaft, and nonlinear bearing operational
TABLE 2: Natural frequency and FRF magnitude comparison.

\begin{tabular}{lcccc}
\hline & Order & Experimentation & Simulation & Error (\%) \\
\hline & 1 & 869.5 & 909 & 4.54 \\
Natural & 2 & 1285 & 1344 & 4.59 \\
frequency $[\mathrm{Hz}]$ & 3 & 2584 & 2477 & 4.14 \\
& 4 & 4348 & 4453 & 2.41 \\
\hline & 1 & 3.25 & 3.07 & 5.53 \\
FRF magnitude & 2 & 4.31 & 4.16 & 3.48 \\
{$\left[\mathrm{~m} / \mathrm{s}^{2} / \mathrm{N}\right]$} & 3 & 6.56 & 6.92 & 5.48 \\
& 4 & 18.48 & 18.13 & 1.89 \\
\hline
\end{tabular}

stiffness, will influence the dynamic performance of SBS that is operating in high-speed field. However, each single highspeed effect differently affects the SBS dynamic performance. Therefore, it is necessary to study these effects one by one. High-speed effects on SBS dynamic performances are studied systematically from the following four aspects.

(1) Only centrifugal effect of shaft is considered.

(2) Only gyroscopic moment effect of shaft is considered.

(3) Only nonlinear bearing operational stiffness is considered.

(4) All of the high-speed effects are considered.

4.1. Centrifugal Effect of Shaft. Based on (11), if shaft speed $\Omega$ is increasing, stiffness of the system will drop due to the term $\Omega^{2}\left[M_{\mathrm{bc}}\right]$, which is so-called spin-soften effect. Thus frequency of SBS will drop too. As indicated in Figure 12, when shaft speed reaches $20000 \mathrm{rpm}$, natural frequencies of the first four normal modes of system drop $6.4 \%, 2.9 \%, 0.8 \%$, and $0.2 \%$, respectively. The simulation also indicates that centrifugal effect of shaft has a greater influence on the lower normal modes than on the higher ones.

4.2. Gyroscopic Moment Effect of Shaft. According to the rotor dynamics [21], if the shaft is in forward whirl situation, then gyroscopic moment lessens the deformation of shaft, which is the equivalent of stiffening the shaft, or, in other 


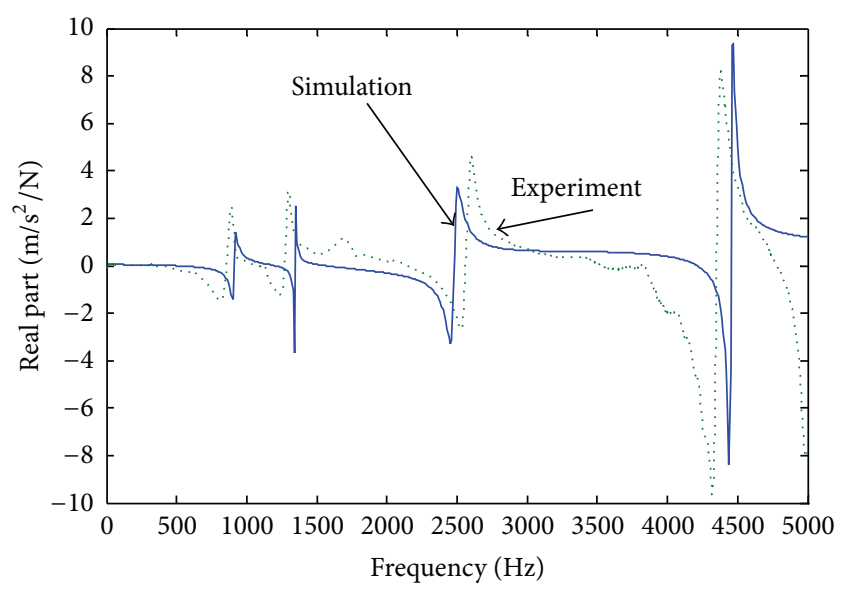

(a) Real part

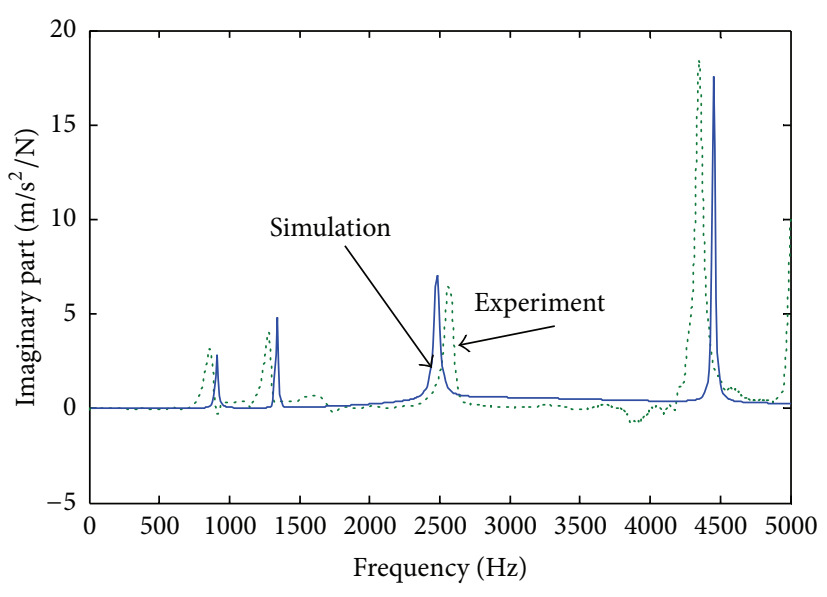

(b) Imaginary part

FIGURE 11: Experimentation versus simulation of $\mathbf{H}_{y y}$ at front tip.

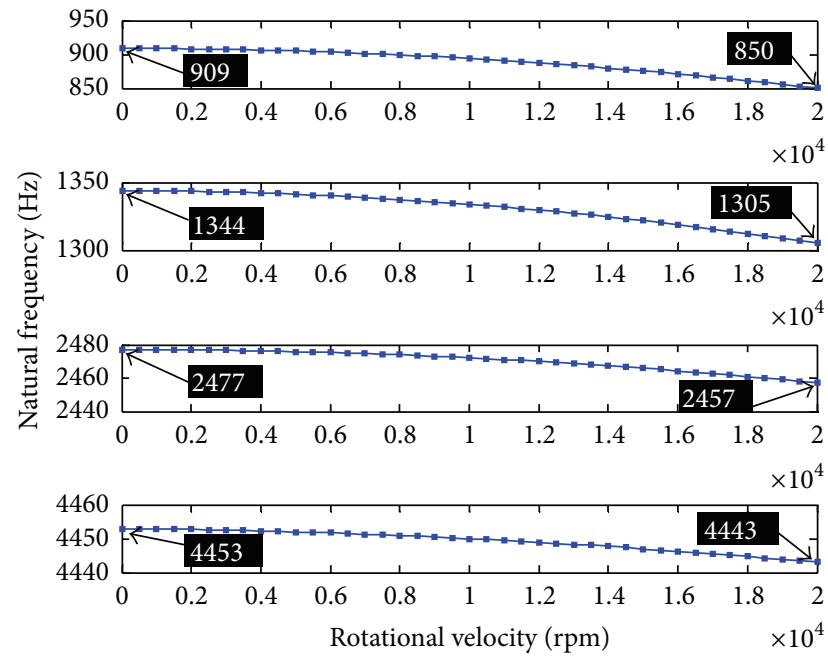

FIGURE 12: Centrifugal force effect of shaft only.

words, natural frequencies of the system increase. Oppositely, if the shaft is in backward whirl situation, natural frequencies of the system decrease. The higher the shaft speed is, the more obvious the gyroscopic moment effect is. This phenomenon theoretically makes every vibration mode split into two modes (see Figure 13). As depicted in Figure 13, gyroscopic moment effect of shaft, similar to centrifugal effect, influences the lower modes greater than the higher ones. Moreover, the gyroscopic moment affects the system more than the centrifugal effect. For instance, at the shaft speed of $20000 \mathrm{rpm}$ and assuming that the shaft is in backward whirl situation, system natural frequencies of the first four normal modes drop $6.8 \%, 8.11 \%, 4.3 \%$, and $0.3 \%$, respectively. Figure 14 shows the variation trends of natural frequencies of the first four normal modes, where both centrifugal effect and gyroscopic moment effect of shaft are considered simultaneously.

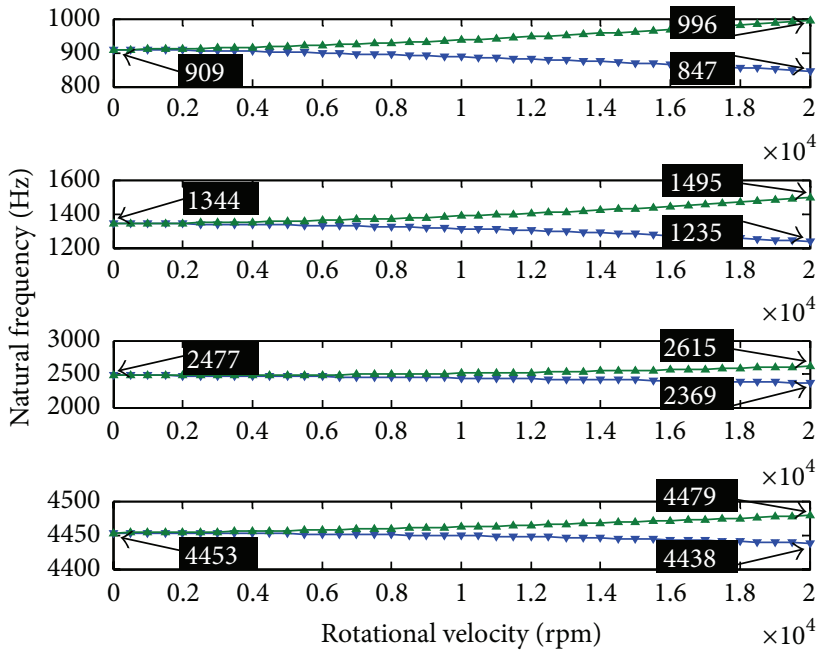

\section{$\triangle$ Forward \\ Backward}

Figure 13: Gyroscopic moment effect of shaft only.

As the damping matrix $[C]$ consists in gyroscopic moment matrices of beam elements, $\left[G_{\mathrm{bm}}\right]$, and disk elements, $\left[G_{\text {disk }}\right]$, in (13), the forward and backward modes of shaft should be reflected in the FRF. Direct FRF $\mathbf{H}_{y y}$ at the front tip is calculated based on the validated FE model in 3.2, and the rotating speed is set as $10000 \mathrm{rpm}$. Three direct FRFs are presented in Figure 15, where the structural damping ratios, $\xi_{\text {system }}$, are set as $0.5 \%, 2 \%$, and $5 \%$, respectively. It is clearly seen that there are two peaks at every normal mode due to the forward mode and the backward mode when the structural damping ration is $0.5 \%$. If the structural damping ratio increases to $2 \%$, magnitudes of two peaks of every normal mode diminish and peaks become closer. If the structural damping reaches $5 \%$, however, the two peaks of each mode can hardly be observed. The simulation indicates 


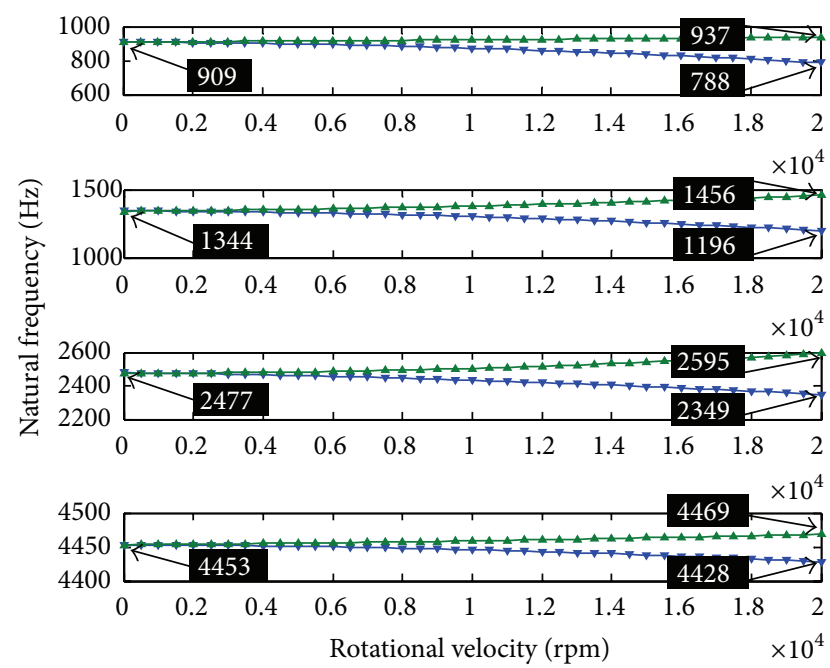

$\triangle$ Forward

$\nabla$ Backward

FIGURE 14: Centrifugal force and gyroscopic moment effects of shaft simultaneously.

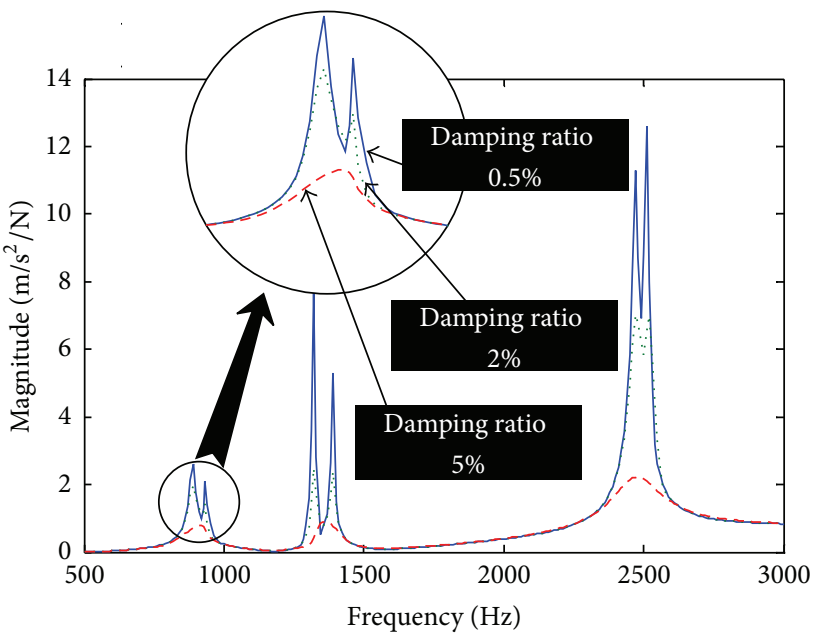

FIGURE 15: Gyroscopic moment effect of shaft on direct FRF $\mathbf{H}_{y y}$ at front tip. Rotational velocity: $10000 \mathrm{rpm}$.

that gyroscopic moment effect becomes less observable if the whole structural damping is quite high.

However, gyroscopic moment effect of shaft influences the cross FRF $\mathbf{H}_{y z}$ at the front tip in a different way. Figure 16 shows that, when system stays in the stationary condition, where the gyroscopic moment term does not exist, the magnitude of $\mathbf{H}_{y z}$ is too tiny to be observed. Once the shaft speed is set as $10000 \mathrm{rpm}$, the magnitude of $\mathbf{H}_{y z}$ is amplified due to the gyroscopic moment effect.

4.3. Nonlinear Operational Stiffness of Angular Contact Ball Bearing. Basic structural parameters of NSK high-speed

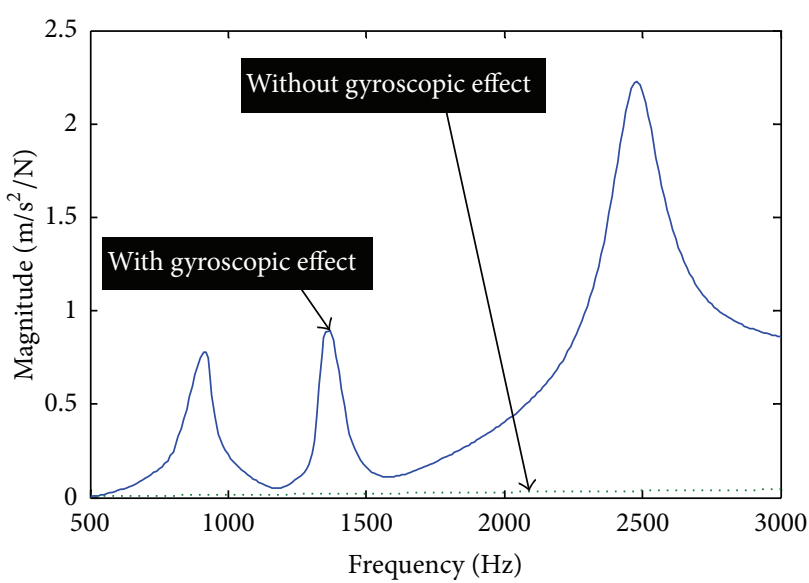

FIgURE 16: Gyroscopic moment effect of shaft on cross FRF $\mathbf{H}_{y z}$ at front tip. Rotational velocity: $10000 \mathrm{rpm}$. Damping ratio: 5\%.

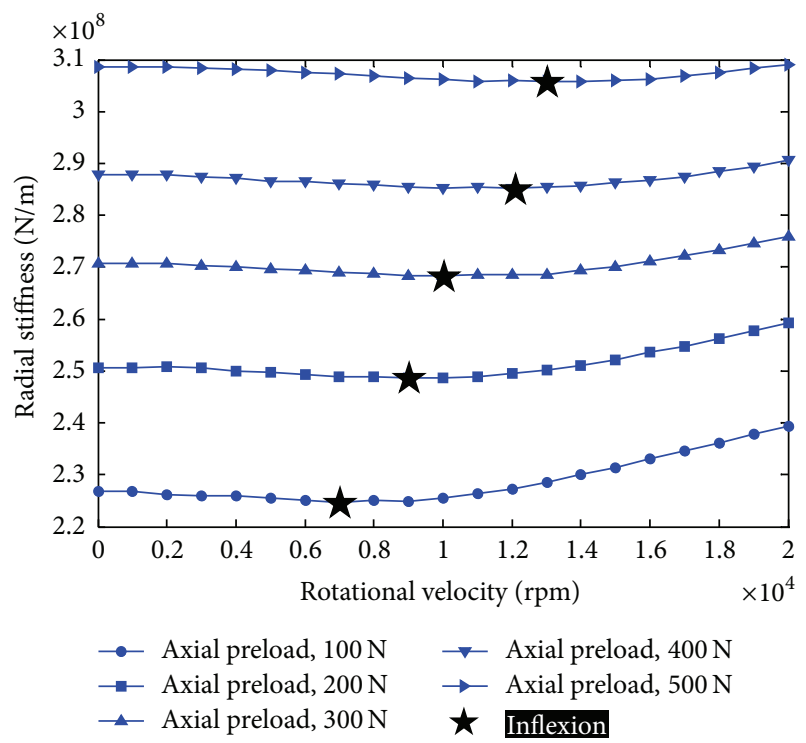

FIGURE 17: Radial operational stiffness of bearing versus various preloads.

angular contact ceramic ball bearings 70BNR10X are illustrated in Table 1. Radial operational stiffness of bearing is calculated based on the contents in 2.1 with respect to different preloads and different rotating speed. Figure 17 shows the calculation results of radial operational stiffness of bearing under different operating conditions. It can be easily seen that, as the bearing rotating speed keeps increasing, radial operational stiffness decreases at first and later on increases evidently.

The nonmonotonic variation trends with respect to rotating speed depicted in Figure 17 is more comprehensive than literature works in which only "soften" phenomena are emphasized but "stiffen" phenomena are neglected. The nonmonotonic variation can be explained as the following statements. First, the centrifugal force acting on the ball becomes greater due to the increasing speed of bearing. Consequently, contact load between ball and inner ring decreases while that between the ball and outer ring increases. 


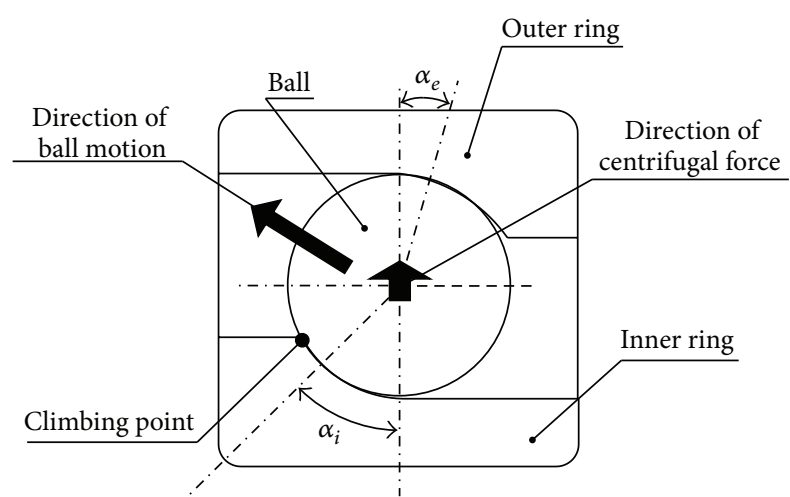

FIGURE 18: Microgeometry of angular contact ball bearing in operational state.

Whereas the radial stiffness, which is the tandem result of contact stiffness, will decrease.

Second, if the bearing speed keeps increasing, the centrifugal force becomes so massive that it makes the ball begin to climb up the inner ring (see motion of ball in Figure 18). This climbing motion increases the contact load between ball and the inner ring dramatically and finally increases the radial stiffness of bearing.

Therefore, with consideration of a wide range of rotating speed, radial operational stiffness of angular ball bearing will not decrease constantly as the speed increases. Once the critical rotating speed is reached, bearing stiffness will increase.

Figure 17 also indicates that inflexion of stiffness-speed curve drifts to higher speed range when the bearing axial preload increases. Thus it is reasonable to hypothesize that, if bearing axial preload increases to a certain degree, inflexion of the stiffness-speed curve will disappear within a particular speed range. In order to check this prediction, radial operational stiffness of 70BNR10X is recalculated according to the three reference preloads [22]: EL (285 N), L $(1330 \mathrm{~N})$, and M $(2568 \mathrm{~N})$. The simulation shown in Figure 19 agrees with the hypothesis.

During the practical assemblage process, axial preload of bearing 70BNR10X is set as EL $(285 \mathrm{~N})$, which is illustrated in 3.1 already. Based on the operational stiffness calculated on the condition with EL preload, the SBS dynamic performance is analyzed without considering the centrifugal effect and gyroscopic moment effect of shaft. Natural frequencies of the first four normal modes have a similar nonmonotonic variation tendency to the bearing stiffness, but, nevertheless, the variation amplitude is extremely tiny as shown in Figure 20. From the analysis above, it is found that, under the EL preload condition, nonlinear operational stiffness plays an insignificant role in the dynamic performance of high-speed running SBS.

4.4. High-Speed Effects on Dynamic Performance of SBS. With the consideration of all the high-speed effects, SBS dynamic performance is simulated. Natural frequencies of the first four normal modes are very different from the circumstance

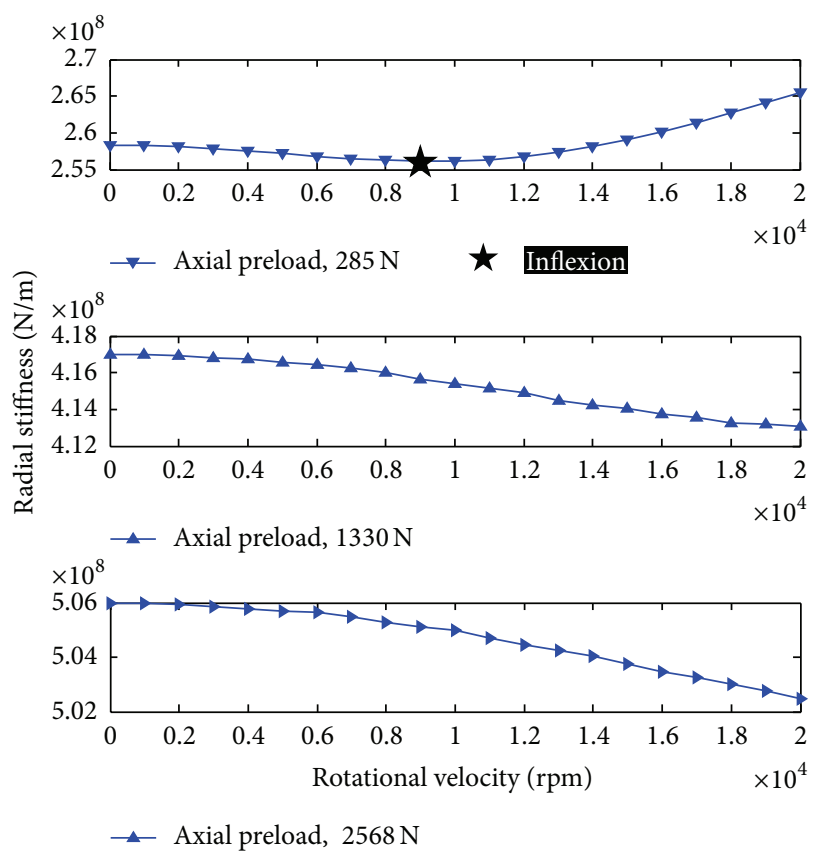

FIGURE 19: Radial operational stiffness of 70BNR10X bearing versus various preloads.
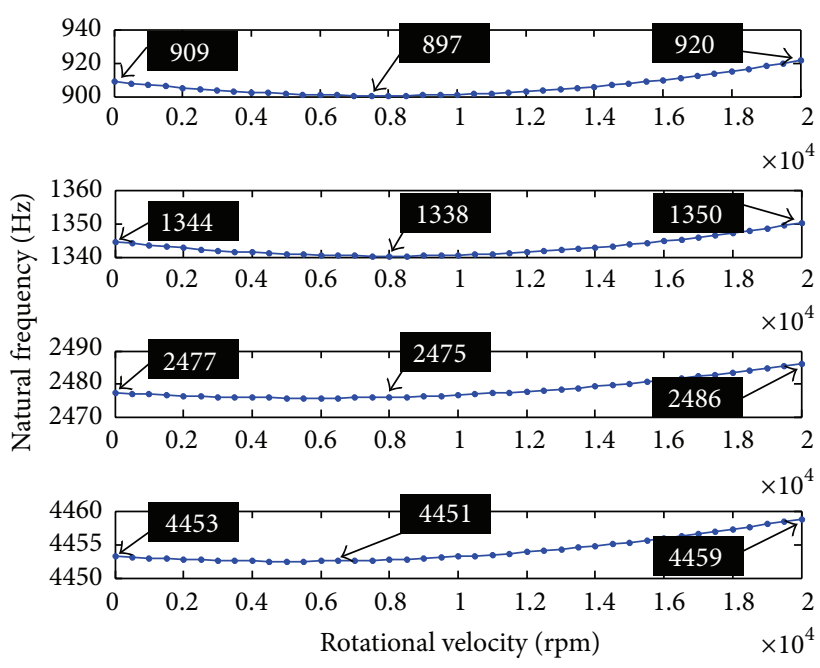

FIGURE 20: Operational stiffness of angular contact ball bearing only.

where only nonlinear bearing operational stiffness is concerned (see Figure 21). It can be concluded by connecting the contents 4.1-4.3 that, under the EL bearing preload condition, high-speed effects of shaft, especially the gyroscopic moment, are the most critical affection factors to the dynamic performance of SBS that runs in the high-speed field.

\section{Conclusion}

Shaft-bearing system dynamic performance is influenced by high-speed effects. This paper presents a dynamic model which can predict the high-speed performance of a SBS running in the high-speed field. The Timoshenko beam 


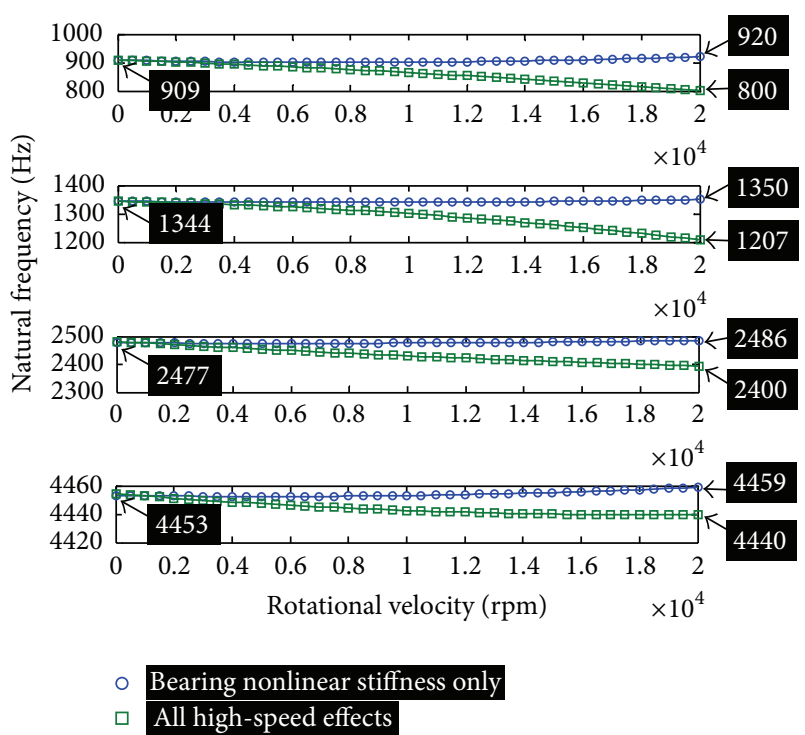

FIGURE 21: High-speed effects of both shaft and bearing versus highspeed effects of bearing only.

theory and FEM are employed to simulate the shaft, housing, and motor stator. Both centrifugal effect and gyroscopic effect are included in creating the beam model. The SBS FE model is formulated by coupling the nonlinear bearing model developed on the basis of the Hertzian contact theory. Idle state of FRF at the front tip is obtained analytically and experimentally to validate that the proposed FE model is reliable for illustrating the SBS dynamic behaviors in static condition. Simulations are conducted to systematically investigate the high-speed effects on the SBS dynamic performance by substituting varying rotational velocities into the FE model. The results show that both centrifugal effect and gyroscopic effect of shaft affect the lower modes more than the higher ones. Furthermore, gyroscopic moment of shaft affects the front tip of cross FRF considerably, yet it can hardly affect the direct FRF because of the structural damping ratio. The nonmonotonic variation of nonlinear bearing stiffness is observed and explained, where the stiffness-speed curve inflexion drifting towards the high-speed range due to the increasing axial preload is hypothesized and proved through the bearing model.

In conclusion, under the EL bearing preload condition, the high-speed effects of shaft have a greater influence on SBS dynamic performance than nonlinear bearing stiffness. Moreover, both "soften" and "stiffen" phenomena exist for the running bearing, which provides a more comprehensive understanding of the bearing nonlinear characteristics. Future study is planned to develop the high-speed experimentation platform, on which the simulation of high-speed effects illustrated in this paper can be verified.

\section{Nomenclature}

Q: Normal load

$k$ : Contact coefficient

$K$ : Contact stiffness $\alpha$ : Contact angle

$f: \quad r / D$

$r$ : Radius of ring curvature

$D:$ Ball diameter

$\omega_{m}:$ Orbital speed of ball

$\omega_{R}$ : Speed of ball about its own axis

$F$ : Force

$M:$ Moment

B: $\quad f_{i}+f_{e}-1$

$\delta$ : Contact deformation or deflection

$M_{g}$ : Gyroscopic moment

$\psi:$ Azimuth

$F_{c}$ : Centrifugal force

$m: \quad$ Ball mass

$d_{m}$ : Pitch diameter

$\omega: \quad$ Shaft speed

$\beta$ : $\quad$ Ball attitude angle

$\Re$ : Radius of locus of ring curvature center

$\theta$ : Angular displacement.

\section{Subscripts}

$\begin{array}{ll}a: & \text { Axial direction } \\ r: & \text { Radial direction } \\ i: & \text { Inner ring } \\ x, y, z: & x, y, z \text { direction } \\ j: & \text { The jth ball } \\ e: & \text { Outer ring. }\end{array}$

\section{Conflict of Interests}

The authors declare that there is no conflict of interests regarding the publication of this paper.

\section{Acknowledgments}

This research is sponsored by National Science and Technology Major Project of China (nos. SK201201A26-01 and 2013ZX04005-012).

\section{References}

[1] A. Palmgren, Ball and Roller Bearing Engineering, SKF industries, Burbank Philadelphia, 3rd edition, 1959.

[2] A. B. Jones, "Ball motion and sliding friction in ball bearings," Journal of Basic Engineering, vol. 3, pp. 1-15, 1959.

[3] A. B. Jones, "A general theory for elastically constrained ball and radial roller bearings under arbitrary load and speed condition," Journal of Basic Engineering, vol. 82, no. 2, pp. 309-320, 1960.

[4] T. A. Harris, Rolling Bearing Analysis, CRC Press, Florida, 5th edition, 2006.

[5] P. K. Gupta, Advanced Dynamics of Rolling Elements, Springer, New York, NY, USA, 1984.

[6] N. T. Liao and J. F. Lin, "A new method for the analysis of deformation and load in a ball bearing with variable contact angle," Journal of Mechanical Design, Transactions of the ASME, vol. 123, no. 2, pp. 304-312, 2001.

[7] I. Zverv, Y.-S. Pyoun, K.-B. Lee, J.-D. Kim, I. Jo, and A. Combs, "An elastic deformation model of high speed spindles built into 
ball bearings," Journal of Materials Processing Technology, vol. 170, no. 3, pp. 570-578, 2005.

[8] Y. Kang, P.-C. Shen, C.-C. Huang, S.-S. Shyr, and Y.-P. Chang, "A modification of the Jones-Harris method for deep-groove ball bearings," Tribology International, vol. 39, no. 11, pp. 1413-1420, 2006.

[9] Y. Kang, C.-C. Huang, C.-S. Lin, P.-C. Shen, and Y.-P. Chang, "Stiffness determination of angular-contact ball bearings by using neural network," Tribology International, vol. 39, no. 6, pp. 461-469, 2006.

[10] J. Jedrzejewski and W. Kwasny, "Modelling of angular contact ball bearings and axial displacements for high-speed spindles," CIRP Annals, Manufacturing Technology, vol. 59, no. 1, pp. 377382, 2010.

[11] Y. Guo and R. G. Parker, "Stiffness matrix calculation of rolling element bearings using a finite element/contact mechanics model," Mechanism and Machine Theory, vol. 51, pp. 32-45, 2012.

[12] H. D. Nelson, "A finite rotating shaft element using Timoshenko Beam Theory," Journal of mechanical design, vol. 102, no. 4, pp. 793-803, 1980.

[13] C.-W. Lin, J. F. Tu, and J. Kamman, "An integrated thermomechanical-dynamic model to characterize motorized machine tool spindles during very high speed rotation," International Journal of Machine Tools and Manufacture, vol. 43, no. 10, pp. 1035-1050, 2003.

[14] A. Ertürk, H. N. Özgüven, and E. Budak, "Analytical modeling of spindle-tool dynamics on machine tools using Timoshenko beam model and receptance coupling for the prediction of tool point FRF,' International Journal of Machine Tools and Manufacture, vol. 46, no. 15, pp. 1901-1912, 2006.

[15] T. L. Schmitz, M. A. Davies, and M. D. Kennedy, “Tool point frequency response prediction for high-speed machining by RCSA," Journal of Manufacturing Science and Engineering, Transactions of the ASME, vol. 123, no. 4, pp. 700-707, 2001.

[16] S. Jiang and S. Zheng, "A modeling approach for analysis and improvement of spindle-drawbar-bearing assembly dynamics," International Journal of Machine Tools and Manufacture, vol. 50, no. 1, pp. 131-142, 2010.

[17] J.-S. Chen and Y.-W. Hwang, "Centrifugal force induced dynamics of a motorized high-speed spindle," International Journal of Advanced Manufacturing Technology, vol. 30, no. 1-2, pp. 10-19, 2006.

[18] E. Wang, B. Wu, Y. Hu et al., "Dynamic parameter identification of tool-spindle interface based on RCSA and Particle Swarm Optimization," Shock and Vibration, vol. 20, no. 1, pp. 69-78, 2013.

[19] J. H. Ginsberg, Mechanical and Structural Vibrations Theory and Applications, China Astronautic Publishing House, 2005.

[20] K.-J. Bathe, Finite Element Procedures, Prentice Hall, Upper Saddle River, New Jersey, NJ, USA, 1982.

[21] J. Vance, F. Zeidan, and B. Murphy, Machinery Vibration and Rotordynamics, John Wiley \& Sons, New Jersey, NJ, USA, 2010.

[22] NSK, Super precision bearings, CAT. No.E1254f 2011 D-2 Printed in Japan NSK Ltd.2003, 2011. 

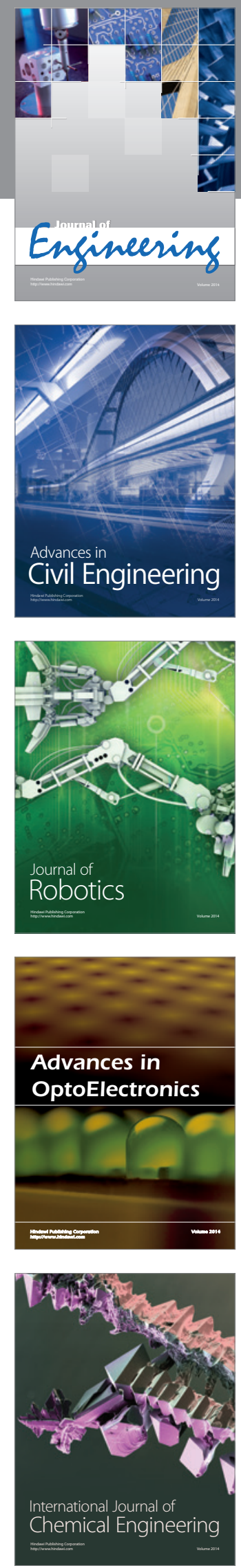

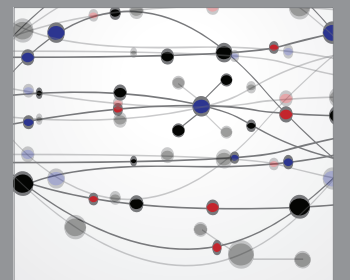

The Scientific World Journal
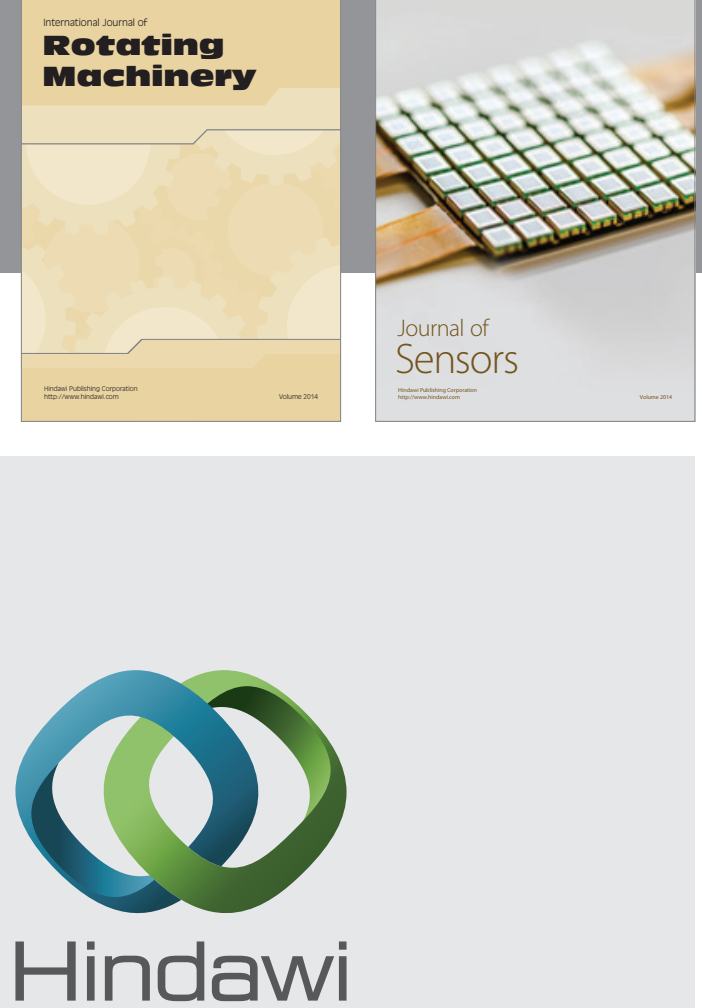

Submit your manuscripts at http://www.hindawi.com
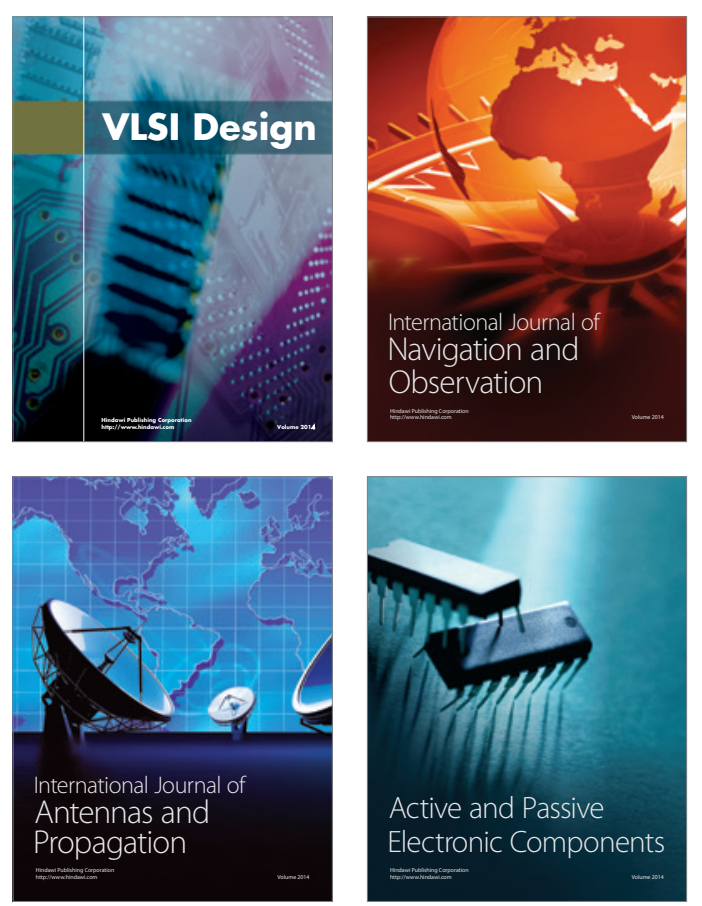
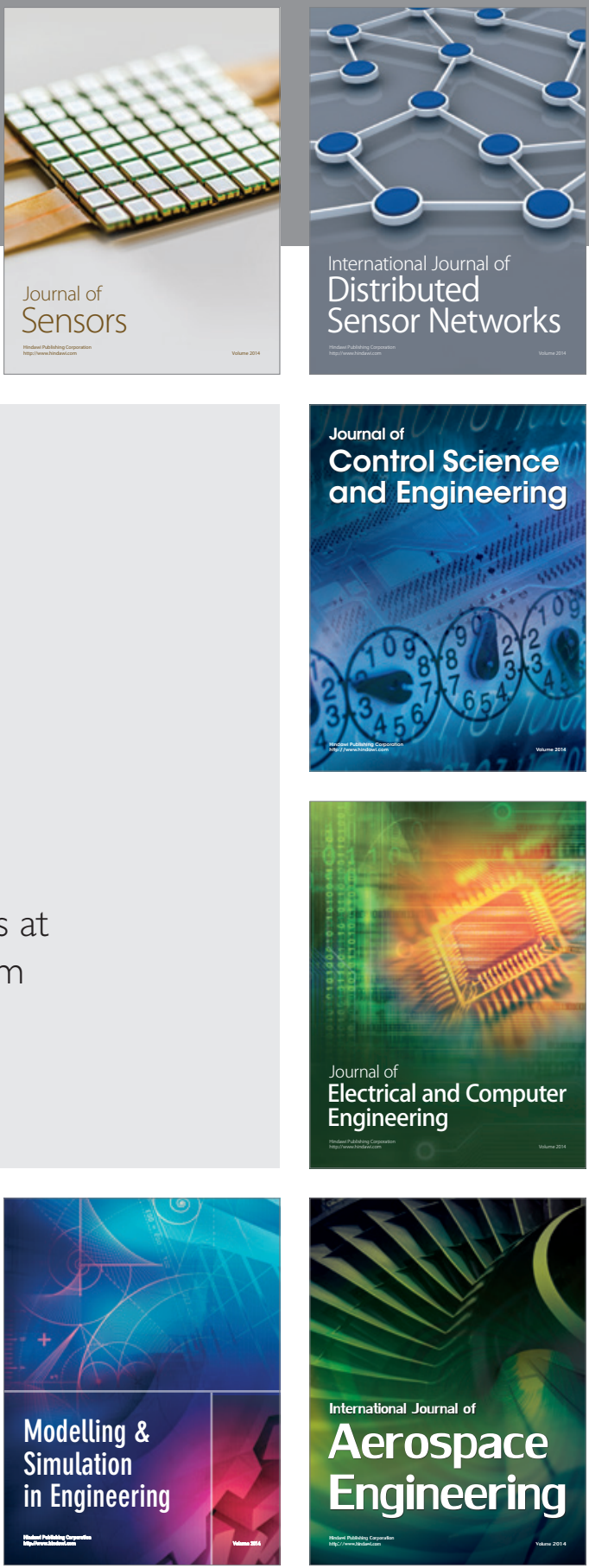

Journal of

Control Science

and Engineering
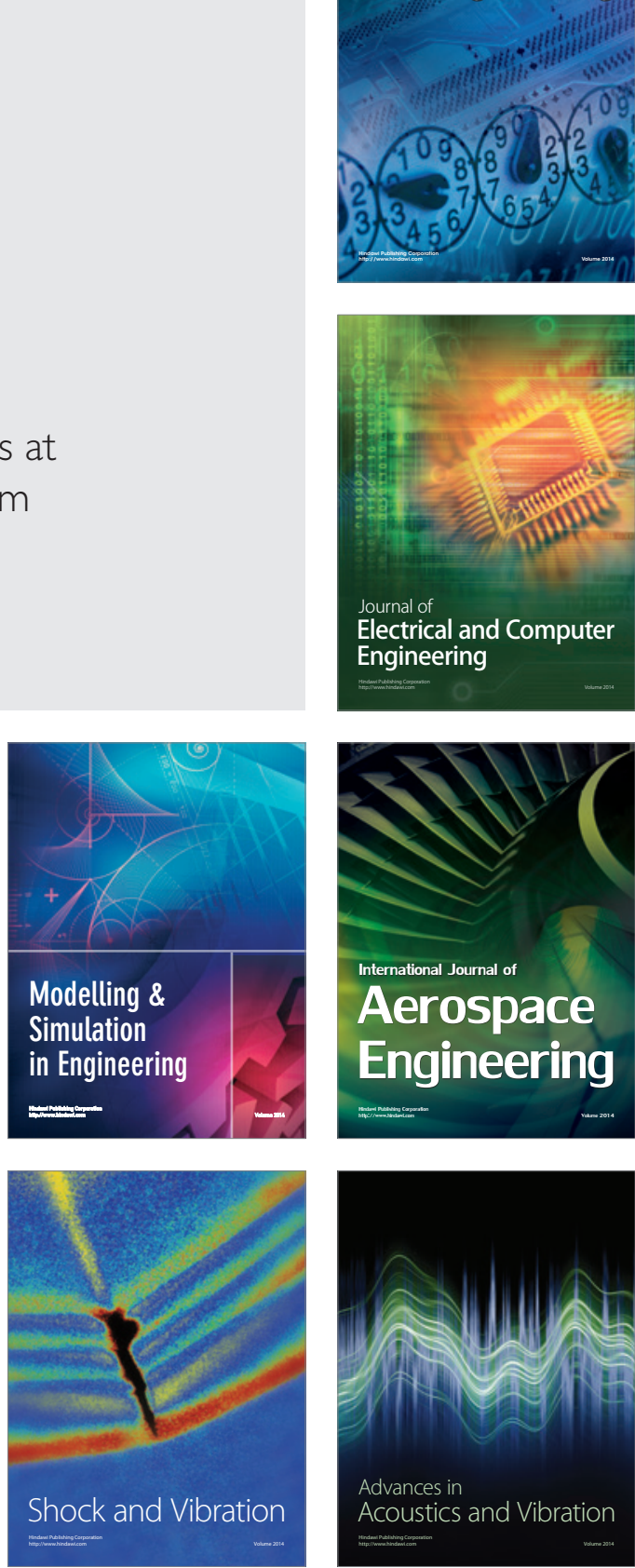